\title{
UNIFORM ASYMPTOTICS FOR DISCOUNTED AGGREGATE CLAIMS IN DEPENDENT RISK MODELS
}

\author{
YANG YANG, * Nanjing Audit University and Southeast University \\ KAIYONG WANG, ${ }^{* *}$ Southeast University \\ DIMITRIOS G. KONSTANTINIDES, ${ }^{* * *}$ University of the Aegean
}

\begin{abstract}
In this paper we consider some nonstandard renewal risk models with some dependent claim sizes and stochastic return, where an insurance company is allowed to invest her/his wealth in financial assets, and the price process of the investment portfolio is described as a geometric Lévy process. When the claim size distribution belongs to some classes of heavy-tailed distributions and a constraint is imposed on the Lévy process in terms of its Laplace exponent, we obtain some asymptotic formulae for the tail probability of discounted aggregate claims and ruin probabilities holding uniformly for some finite or infinite time horizons.
\end{abstract}

Keywords: Discounted aggregate claim; dependence; Lévy process; consistently varying tail; dominatedly varying tail; long tail; uniformity

2010 Mathematics Subject Classification: Primary 91B30

Secondary 60G51; 60K05

\section{Introduction}

We consider a nonstandard renewal risk model in which successive claim sizes $\left\{X_{n}, n \geq 1\right\}$ form a sequence of identically distributed but not necessarily independent nonnegative random variables (RVs) with common distribution $F$, and the interarrival times $\left\{\theta_{n}, n \geq 1\right\}$ form another sequence of independent and identically distributed (i.i.d.) nonnegative RVs, which are independent of $\left\{X_{n}, n \geq 1\right\}$. We suppose that the claim arrival times $\tau_{n}=\sum_{k=1}^{n} \theta_{k}, n \geq 1$, constitute a renewal counting process

$$
N(t)=\sup \left\{n \geq 0: \tau_{n} \leq t\right\}, \quad t \geq 0,
$$

which represents the number of claims up to time $t$, with a finite mean function $\lambda(t)=$ $\mathbb{E}[N(t)] \rightarrow \infty$ as $t \rightarrow \infty$. Suppose that the insurer is allowed to make risk-free and risky investments. The price process of the investment portfolio is described as a geometric Lévy process $\left\{\mathrm{e}^{R_{t}}, t \geq 0\right\}$ with $\left\{R_{t}, t \geq 0\right\}$ being a Lévy process, which starts from 0 and has independent and stationary increments. This assumption on price processes is widely used in mathematical finance. The reader is referred to [4], [11], [15], [17], [18], [19], [20], [24], [26], and [27], among others.

Received 17 May 2013; revision received 8 August 2013.

* Postal address: School of Mathematics and Statistics, Nanjing Audit University, Nanjing, 210029, China.

Email address: yyangmath@gmail.com

** Postal address: Department of Mathematics, Southeast University, Nanjing, 210096, China.

*** Postal address: Department of Mathematics, University of the Aegean, Karlovassi, GR-83 200 Samos, Greece.

Email address: konstant@aegean.gr 
As usual, we assume that $\left\{X_{n}, n \geq 1\right\},\left\{\theta_{n}, n \geq 1\right\}$, and $\left\{R_{t}, t \geq 0\right\}$ are mutually independent. The discounted aggregate claims up to time $t \geq 0$ can be expressed as

$$
D(t)=\sum_{k=1}^{\infty} X_{k} \mathrm{e}^{-R_{\tau_{k}}} \mathbf{1}_{\left\{\tau_{k} \leq t\right\}},
$$

where $\mathbf{1}_{A}$ denotes the indicator function of an event $A$. Then, for any $t \geq 0$, the discounted value of the surplus process with stochastic return on investments of an insurance company is described as

$$
U(t)=x+\int_{0-}^{t} c(s) \mathrm{e}^{-R_{s}} \mathrm{~d} s-D(t),
$$

where $x \geq 0$ is the initial risk reserve of the insurance company and $c(t)$ denotes the density function of premium income at time $t$. Throughout the paper, we assume that the premium density function $c(t)$ is bounded, i.e. $0 \leq c(t) \leq M$ for some constant $M>0$ and all $t \geq 0$. In this way, in the above renewal risk model, for any $t \geq 0$, the finite-time ruin probability can be defined as

$$
\psi(x, t)=\mathbb{P}\left(\inf _{s \in[0, t]} U(s)<0 \mid U(0)=x\right)
$$

and the infinite-time ruin probability as

$$
\psi(x, \infty)=\mathbb{P}\left(\inf _{s \geq 0} U(s)<0 \mid U(0)=x\right) .
$$

In the present paper we shall investigate the asymptotics for the tail probability of discounted aggregate claims or ruin probabilities, holding uniformly for all $t$, such that $\lambda(t)$ is positive. For this purpose, as in [22], define $\Lambda=\{t: 0<\lambda(t) \leq \infty\}=\left\{t: \mathbb{P}\left(\theta_{1} \leq t\right)>0\right\}$ with $\underline{t}=\inf \{t: \lambda(t)>0\}=\inf \left\{t: \mathbb{P}\left(\theta_{1} \leq t\right)>0\right\}$. Clearly,

$$
\Lambda= \begin{cases}{[\underline{t}, \infty]} & \text { if } \mathbb{P}\left(\theta_{1}=\underline{t}\right)>0, \\ (\underline{t}, \infty] & \text { if } \mathbb{P}\left(\theta_{1}=\underline{t}\right)=0 .\end{cases}
$$

For the above risk model with a constant premium rate $c>0$ (i.e. $c(t)=c$ for all $t>0$ ) and a constant interest force $\delta>0$ (e.g. $R_{t}=\delta t$ for all $t>0$ ), for which the claim sizes $\left\{X_{n}, n \geq 1\right\}$ and the interarrival times $\left\{\theta_{n}, n \geq 1\right\}$ are i.i.d. RVs, some earlier works on ruin probabilities can be found in [1], [10], [12], [13], and [21], among others; Tang [22] and Hao and Tang [8] derived some uniform results in $\Lambda$. Ruin probabilities have also been investigated by many researchers for claim sizes $\left\{X_{n}, n \geq 1\right\}$ that follow a certain dependence structure. Chen and $\mathrm{Ng}$ [5] considered a dependent risk model, where the claim sizes are pairwise negatively quadrant dependent (see the definition in Section 2.1) with common distribution $F$, belonging to the class of extended regularly varying distributions. They obtained the following asymptotics for the infinite-time ruin probability:

$$
\psi(x, \infty) \sim \int_{0-}^{\infty} \bar{F}\left(x \mathrm{e}^{\delta u}\right) \lambda(\mathrm{d} u) .
$$

Furthermore, assuming some restrictive dependence structure and heavy-tailed claim sizes, Yang and Wang [25] showed that the relation

$$
\psi(x, t) \sim \int_{0-}^{t} \bar{F}\left(x \mathrm{e}^{\delta u}\right) \lambda(\mathrm{d} u)
$$

holds either for each fixed $t \in \Lambda$ or uniformly for all $t \in \Lambda$. 
In the two recent interesting papers by Tang et al. [24] and Li [15] the above renewal risk model with stochastic return was investigated, with the price process of the investment portfolio being a geometric Lévy process under independence and dependence structures, respectively. We note that Tang et al. [24] investigated the independent renewal risk model in which the claim sizes $\left\{X_{n}, n \geq 1\right\}$ and the interarrival times $\left\{\theta_{n}, n \geq 1\right\}$ are two sequences of i.i.d. nonnegative RVs that are mutually independent, whereas Li [15] considered a more general time-dependent renewal risk model in which $\left\{\left(X_{n}, \theta_{n}\right), n \geq 1\right\}$ are assumed to be i.i.d. random vectors with a certain dependence existing between $X_{n}$ and $\theta_{n}$ for each fixed $n$. Motivated by these two papers, the main goal of this paper is to establish some asymptotic formulae for the tail probability of discounted aggregate claims and ruin probabilities, holding uniformly for some finite or infinite time horizons, under the conditions that the claim sizes $\left\{X_{n}, n \geq 1\right\}$ are dependent and have common distribution belonging to the class of long and dominatedly varying tailed distributions or to the class of consistently varying tailed distributions.

The rest of the paper is organized as follows. In Section 2, after introducing some preliminaries, we present the four main results on the uniform asymptotics for the tail probability of discounted aggregate claims and ruin probabilities. In Sections 3 and 4 we prove these results after preparing a series of lemmas.

\section{Preliminaries and main results}

Hereafter, all limit relationships hold for $x$ tending to $\infty$, unless stated otherwise. For two positive functions $a(x)$ and $b(x)$, we write $a(x) \sim b(x)$ if $\lim a(x) / b(x)=1 ; a(x) \lesssim b(x)$ or $b(x) \gtrsim a(x)$ if $\lim \sup a(x) / b(x) \leq 1 ; a(x)=o(b(x))$ if $\lim a(x) / b(x)=0 ; a(x)=O(b(x))$ if $\lim \sup a(x) / b(x)<\infty$; and $a(x) \asymp b(x)$ if $a(x)=O(b(x))$ and $b(x)=O(a(x))$. Furthermore, for two positive bivariate functions $a(x, t)$ and $b(x, t)$, we write $a(x, t) \sim b(x, t)$ uniformly for all $t$ in a nonempty set $A$ if

$$
\lim _{x \rightarrow \infty} \sup _{t \in A}\left|\frac{a(x, t)}{b(x, t)}-1\right|=0,
$$

and $a(x, t) \lesssim b(x, t)$ or $b(x, t) \gtrsim a(x, t)$ uniformly for all $t \in A$ if

$$
\limsup _{x \rightarrow \infty} \sup _{t \in A} \frac{a(x, t)}{b(x, t)} \leq 1 .
$$

\subsection{Dependence structures}

Since we are interested in actual dependent risk models, we start by introducing some dependence structures. A sequence of RVs $\left\{\xi_{n}, n \geq 1\right\}$ is said to be pairwise negatively quadrant dependent (NQD) if, for any $i \neq j \geq 1$ and real $x, y$,

$$
\mathbb{P}\left(\xi_{i}>x, \xi_{j}>y\right) \leq \mathbb{P}\left(\xi_{i}>x\right) \mathbb{P}\left(\xi_{j}>y\right) .
$$

The pairwise NQD structure was introduced by Lehmann [14], and is weaker and more verifiable than the commonly used notions of the upper/lower negative dependence (see [3]), and the negative association (see [9]). A more general dependence structure, namely the upper tail asymptotic independence structure, was proposed by Maulik and Resnick [16]. A sequence of $\operatorname{RVs}\left\{\xi_{n}, n \geq 1\right\}$ is said to be upper tail asymptotic independent (UTAI) if $\mathbb{P}\left(\xi_{n}>x\right)>0$ for all $x \in \mathbb{R}$ and $n \geq 1$, and

$$
\lim _{\min \{x, y\} \rightarrow \infty} \mathbb{P}\left(\xi_{i}>x \mid \xi_{j}>y\right)=0 \quad \text { for any } i \neq j \geq 1 .
$$

Clearly, if a sequence of RVs is pairwise NQD then it is also UTAI. 


\subsection{Some classes of heavy-tailed distributions}

We shall restrict the claim size distribution $F$ to some class of heavy-tailed distributions, whose moment generating functions do not exist. An important class of heavy-tailed distributions is $\mathscr{D}$, which consists of all distributions $F=1-\bar{F}$ with dominated variation. A distribution $F$ on $\mathbb{R}$ belongs to the class $\mathcal{D}$ if $\lim \sup \bar{F}(x y) / \bar{F}(x)<\infty$ for any $0<$ $y<1$. A slightly smaller class is $\mathcal{C}$ of consistently varying distributions. A distribution $F$ on $\mathbb{R}$ belongs to the class $\mathcal{C}$ if $\lim _{y \uparrow 1} \lim \sup _{x \rightarrow \infty} \bar{F}(x y) / \bar{F}(x)=1$. Closely related is a wider class $\mathcal{L}$ of long-tailed distributions. A distribution $F$ on $\mathbb{R}$ belongs to the class $\mathcal{L}$ if $\lim \bar{F}(x+y) / \bar{F}(x)=1$ for any $y \in \mathbb{R}$. There are some other heavy-tailed subclasses, the class $\operatorname{ERV}(-\alpha,-\beta)$ of distributions with extended regularly varying tails, and the class $\mathcal{R}_{-\alpha}$ of distributions with regularly varying tails. A distribution $F$ on $\mathbb{R}$ belongs to the class $\operatorname{ERV}(-\alpha,-\beta)$ if there exist some constants $0<\alpha \leq \beta<\infty$ such that $y^{-\beta} \leq \liminf \bar{F}(x y) / \bar{F}(x) \leq$ $\lim \sup \bar{F}(x y) / \bar{F}(x) \leq y^{-\alpha}$ for any $y \geq 1$. If $\alpha=\beta, F$ belongs to the class $\mathcal{R}_{-\alpha}$.

For a distribution $F$ on $[0, \infty)$, denote its upper Matuszewska index by

$$
J_{F}^{+}=-\lim _{y \rightarrow \infty} \frac{\log \bar{F}_{*}(y)}{\log y} \quad \text { with } \quad \bar{F}_{*}(y):=\liminf _{x \rightarrow \infty} \frac{\bar{F}(x y)}{\bar{F}(x)} \quad \text { for } y>1 .
$$

It follows from the presented definitions that the assertions

$$
F \in \mathscr{D}, \quad \bar{F}_{*}(y)>0 \quad \text { for some } y>1, \quad J_{F}^{+}<\infty
$$

are equivalent (for details, see [2]).

Lemma 2.1. ([2, Proposition 2.2.1].) For a distribution $F \in \mathscr{D}$ on $[0, \infty)$ and any $p>J_{F}^{+}$, there exist positive constants $C_{1}$ and $D_{1}$ such that the inequality

$$
\frac{\bar{F}(y)}{\bar{F}(x)} \leq C_{1}\left(\frac{y}{x}\right)^{-p}
$$

holds for all $x \geq y \geq D_{1}$.

The next lemma follows immediately from Lemma 2.1 (see Lemma 3.5 of [23]).

Lemma 2.2. For a distribution $F \in \mathscr{D}$ on $[0, \infty)$ and any $p>J_{F}^{+}$, the asymptotics $x^{-p}=$ $o(\bar{F}(x))$ hold.

\subsection{Main results}

Suppose that the Lévy process $\left\{R_{t}, t \geq 0\right\}$ in (1.1) is right continuous with left limits. Let $\mathbb{E}\left[R_{1}\right]>0$, so that $R_{t}$ drifts to $\infty$ almost surely as $t \rightarrow \infty$. The Laplace exponent for the Lévy process $\left\{R_{t}, t \geq 0\right\}$ is defined as

$$
\phi(z)=\log \mathbb{E}\left[\mathrm{e}^{-z R_{1}}\right], \quad z \in \mathbb{R} .
$$

If $\phi(z)$ is finite then it holds for any $t \geq 0$ that

$$
\mathbb{E}\left[\mathrm{e}^{-z R_{t}}\right]=\mathrm{e}^{t \phi(z)}<\infty
$$

(see, e.g. [7, Proposition 3.14]).

To simplify the discussion, we assume throughout the paper that $\underline{t}=0$. For any $T \in \Lambda$ and $\epsilon \in \Lambda$, set $\Lambda^{T}=[0, T]$ and $\Lambda_{\epsilon}=[\epsilon, \infty]$. Note that $\epsilon$ can be chosen to be 0 if $\mathbb{P}\left(\theta_{1}=0\right)>0$. 
We are now ready to state the main results of this paper. The first two results below assume UTAI claim sizes, requiring that the Lévy process $\left\{R_{t}, t \geq 0\right\}$ is almost surely nonnegative, which means that the insurer invests her/his wealth only in a risk-free market. We obtain two uniform asymptotic formulae for the tail probability of discounted aggregate claims and ruin probabilities, corresponding to when the claim sizes have either long and dominatedly varying tails or consistently varying tails.

Theorem 2.1. In the nonstandard renewal risk model described in Section 1, assume that the claim sizes $\left\{X_{n}, n \geq 1\right\}$ are UTAI nonnegative $R V$ s with common distribution $F \in \mathcal{L} \cap \mathcal{D}$. If $R_{t} \geq 0$ almost surely for any $t \geq 0$ then, for any fixed $T>0$,

$$
\mathbb{P}(D(t)>x) \sim \int_{0-}^{t} \mathbb{P}\left(X_{1} \mathrm{e}^{-R_{s}}>x\right) \lambda(\mathrm{d} s)
$$

holds uniformly for all $t \in \Lambda^{T}$.

Corollary 2.1. Under the conditions of Theorem 2.1, if $F \in \mathcal{C}$ then, for any fixed $T>0$,

$$
\psi(x, t) \sim \int_{0-}^{t} \mathbb{P}\left(X_{1} \mathrm{e}^{-R_{s}}>x\right) \lambda(\mathrm{d} s)
$$

holds uniformly for all $t \in \Lambda^{T}$.

Our next two results restrict the claim sizes to be pairwise NQD RVs with extended regularly varying tails, but allow the insurer to make risk-free and risky investments. This means that the Lévy process $\left\{R_{t}, t \geq 0\right\}$ can be real valued.

Theorem 2.2. In the nonstandard renewal risk model described in Section 1, assume that the claim sizes $\left\{X_{n}, n \geq 1\right\}$ are pairwise $N Q D$ nonnegative $R V$ s with common distribution $F \in \operatorname{ERV}(-\alpha,-\beta), 0<\alpha \leq \beta<\infty$. If $\phi(r)<0$ for some $r>\beta$ then (2.1) holds uniformly for all $t \in \Lambda_{\epsilon}$.

Corollary 2.2. Under the conditions of Theorem 2.2, (2.2) holds uniformly for all $t \in \Lambda_{\epsilon}$.

\section{Proof of Theorem 2.1}

Before proving our first two results, we cite two lemmas. The following lemma is due to Tang [22].

Lemma 3.1. For the renewal counting process $\{N(t), t \geq 0\}$ described in Section 1, any $v>0$, and any fixed $T>0$, it holds that

$$
\lim _{x \rightarrow \infty} \sup _{t \in \Lambda^{T}} \lambda^{-1}(t) \mathbb{E}\left[N^{v}(t) \mathbf{1}_{\{N(t)>x\}}\right]=0 .
$$

The second lemma was proved in [16].

Lemma 3.2. Consider the exponential functional of the Lévy process $\left\{R_{t}, t \geq 0\right\}$ defined as $Z=\int_{0}^{\infty} \mathrm{e}^{-R_{s}} \mathrm{~d}$ s. For every $v>0$ satisfying $\phi(v)<0$, it holds that $\mathbb{E}\left[Z^{v}\right]<\infty$.

Proof of Theorem 2.1. Since the positive Lévy process (i.e. a subordinator) $\left\{R_{t}, t \geq 0\right\}$ has nondecreasing paths, it holds uniformly for all $t \in \Lambda^{T}$ that

$$
\int_{0-}^{t} \mathbb{P}\left(X_{1} \mathrm{e}^{-R_{s}}>x\right) \lambda(\mathrm{d} s) \geq \mathbb{P}\left(X_{1} \mathrm{e}^{-R_{T}}>x\right) \lambda(t) \asymp \bar{F}(x) \lambda(t),
$$


where the second step follows from Theorem 3.3(iv) of [6] and $F \in \mathscr{D}$. Hence, there exists some positive constant $C_{2}$, such that, for sufficiently large $x$ and all $t \in \Lambda^{T}$,

$$
\int_{0-}^{t} \mathbb{P}\left(X_{1} \mathrm{e}^{-R_{s}}>x\right) \lambda(\mathrm{d} s) \geq C_{2} \bar{F}(x) \lambda(t)
$$

For any integer $m \geq 1, t \in \Lambda^{T}$, and $x>0$,

$$
\mathbb{P}(D(t)>x)=\left(\sum_{n=1}^{m}+\sum_{n=m+1}^{\infty}\right) \mathbb{P}\left(\sum_{k=1}^{n} X_{k} \mathrm{e}^{-R_{\tau_{k}}}>x, N(t)=n\right)=: I_{1}+I_{2}
$$

For $I_{2}$, as $R_{s} \geq 0$ almost surely for all $s \geq 0$, and by Lemma 2.1, Markov's inequality, and Lemma 2.2, it holds uniformly for all $t \in \Lambda^{T}$ that

$$
\begin{aligned}
I_{2} & \leq\left(\sum_{m<n \leq x / D_{1}}+\sum_{n>x / D_{1}}\right) \mathbb{P}\left(\sum_{k=1}^{n} X_{k}>x\right) \mathbb{P}(N(t)=n) \\
& \leq \sum_{m<n \leq x / D_{1}} n \bar{F}\left(\frac{x}{n}\right) \mathbb{P}(N(t)=n)+\mathbb{P}\left(N(t)>\frac{x}{D_{1}}\right) \\
& \leq C_{1} \bar{F}(x) \sum_{m<n \leq x / D_{1}} n^{p+1} \mathbb{P}(N(t)=n)+\left(\frac{x}{D_{1}}\right)^{-(p+1)} \mathbb{E}\left[N^{p+1}(t) \mathbf{1}_{\left\{N(t)>x / D_{1}\right\}}\right] \\
& \lesssim C_{1} \bar{F}(x) \mathbb{E}\left[N^{p+1}(t) \mathbf{1}_{\{N(t)>m\}}\right]
\end{aligned}
$$

for some $p>J_{F}^{+}$. Hence, from (3.1), (3.2), and Lemma 3.1, we obtain

$$
\begin{aligned}
\lim _{m \rightarrow \infty} \limsup _{x \rightarrow \infty} \sup _{t \in \Lambda^{T}} \frac{I_{2}}{\int_{0-}^{t} \mathbb{P}\left(X_{1} \mathrm{e}^{-R_{s}}>x\right) \lambda(\mathrm{d} s)} & \leq \frac{C_{1}}{C_{2}} \lim _{m \rightarrow \infty} \sup _{t \in \Lambda^{T}} \lambda^{-1}(t) \mathbb{E}\left[N^{p+1}(t) \mathbf{1}_{\{N(t)>m\}}\right] \\
& =0
\end{aligned}
$$

We mainly deal with $I_{1}$. Let $H\left(y_{1}, \ldots, y_{n+1}\right)$ be the joint distribution of the random vector $\left(\tau_{1}, \ldots, \tau_{n+1}\right), n \geq 1$. Clearly, for $1 \leq n \leq m, t \in \Lambda^{T}$, and $x>0$,

$$
\begin{aligned}
& \mathbb{P}\left(\sum_{k=1}^{n} X_{k} \mathrm{e}^{\left.-R_{\tau_{k}}>x, N(t)=n\right)}\right. \\
& \quad=\int_{\left\{0 \leq s_{1} \leq \cdots \leq s_{n} \leq t, s_{n+1}>t\right\}} \mathbb{P}\left(\sum_{k=1}^{n} X_{k} \mathrm{e}^{-R_{s_{k}}>x}\right) H\left(\mathrm{~d} s_{1}, \ldots, \mathrm{d} s_{n+1}\right) .
\end{aligned}
$$

Similarly to (3.1), there exist some $0<C_{3}<1$ and large $x_{1}>0$, both depending only on $F$, such that, for all $1 \leq k \leq n$ and $0 \leq s_{k} \leq t \leq T$, when $x \geq x_{1}$, it holds that

$$
\mathbb{P}\left(X_{k} \mathrm{e}^{-R_{s_{k}}}>x\right) \geq C_{3} \bar{F}(x) .
$$

Next, we aim to show that, for some $\Delta>0$, which can be arbitrarily small, there exists some $\tilde{x}$, depending only on $F, \Delta$, and $n$, such that, for all $x \geq \tilde{x}$ and $0 \leq s_{1} \leq \cdots \leq s_{n} \leq t \leq T$, 
$1 \leq n \leq m$,

$$
\begin{aligned}
(1-\Delta) \sum_{k=1}^{n} \mathbb{P}\left(X_{k} \mathrm{e}^{-R_{s_{k}}}>x\right) & \leq \mathbb{P}\left(\sum_{k=1}^{n} X_{k} \mathrm{e}^{-R_{s_{k}}}>x\right) \\
& \leq(1+\Delta) \sum_{k=1}^{n} \mathbb{P}\left(X_{k} \mathrm{e}^{-R_{s_{k}}}>x\right) .
\end{aligned}
$$

For any $\varepsilon \in\left(0, \frac{1}{2}\right)$, as $\mathbb{P}\left(\sup _{s \in[0, T]} R_{s}=\infty\right)=0$, there exists a $\delta=\delta(\varepsilon) \in(0,1)$ such that

$$
\mathbb{P}\left(\sup _{s \in[0, T]} R_{S}<-\log \delta\right) \geq 1-\varepsilon
$$

which implies that, for all $1 \leq k \leq n, 0 \leq s_{k} \leq t \leq T$, and $x>0$,

$$
\begin{aligned}
\mathbb{P}\left(X_{k} \mathrm{e}^{\left.-R_{s_{k}}>x\right)}\right. & \geq \int_{\delta}^{1} \bar{F}\left(\frac{x}{u}\right) \mathbb{P}\left(\mathrm{e}^{-R_{s_{k}}} \in \mathrm{d} u\right) \\
& \geq \bar{F}\left(\frac{x}{\delta}\right) \mathbb{P}\left(\sup _{s \in[0, T]} R_{s}<-\log \delta\right) \\
& \geq(1-\varepsilon) \bar{F}\left(\frac{x}{\delta}\right) .
\end{aligned}
$$

As $F \in \mathcal{L}$, there exists some positive, increasing, and slowly varying function $l(x) \uparrow \infty$ such that $l(x) / x \rightarrow 0$ and, for any fixed constant $K$,

$$
\bar{F}(x-K l(x)) \sim \bar{F}(x),
$$

which implies that, for the above $\varepsilon>0$, there exists some $x_{2} \geq x_{1}$, depending only on $F$ and $\varepsilon$, such that, for all $x \geq x_{2}$,

$$
\bar{F}(x-l(x)) \leq \bar{F}\left(x-\frac{l(x)}{\delta}\right) \leq(1+\varepsilon) \bar{F}(x) .
$$

Since $X_{1}, \ldots, X_{n}$ are UTAI and by $F \in \mathscr{D}$, there exists some $x_{3} \geq x_{2}$, depending only on $F$, $\varepsilon$, and $n$, such that, for all $x \geq x_{3}$ and all $1 \leq i \neq j \leq n(1 \leq n \leq m)$,

$$
\mathbb{P}\left(X_{i}>x, X_{j}>x\right) \leq \mathbb{P}\left(X_{i}>\frac{l(x)}{n-1}, X_{j}>\frac{x}{n}\right) \leq \frac{\varepsilon}{n} C_{3} \bar{F}(x) .
$$

Let $\tilde{x}=\max \left\{x_{3}, D_{1} /(1-\delta)\right\}$, which also depends only on $F, \varepsilon$, and $n$. Then, for $x \geq \tilde{x}$, it holds that $x-l(x) \geq(1-\delta) x$ and, for some $p>J_{F}^{+}$,

$$
\frac{\bar{F}((1-\delta) x)}{\bar{F}(x)} \leq C_{1}(1-\delta)^{-p}
$$

together with (3.10) and (3.11). For the lower bound of (3.6), by the Bonferroni inequality, (3.11), and (3.5) we have, for all $x \geq \tilde{x}$ and $0 \leq s_{k} \leq t \leq T, 1 \leq k \leq n$,

$$
\begin{aligned}
\mathbb{P}\left(\sum_{k=1}^{n} X_{k} \mathrm{e}^{-R_{s_{k}}}>x\right) & \geq \sum_{k=1}^{n} \mathbb{P}\left(X_{k} \mathrm{e}^{-R_{s_{k}}}>x\right)-\sum_{1 \leq i<j \leq n} \mathbb{P}\left(X_{i}>x, X_{j}>x\right) \\
& \geq(1-\varepsilon) \sum_{k=1}^{n} \mathbb{P}\left(X_{k} \mathrm{e}^{-R_{s_{k}}}>x\right) .
\end{aligned}
$$


For the upper bound of (3.6),

$$
\begin{aligned}
\mathbb{P}\left(\sum_{k=1}^{n} X_{k} \mathrm{e}^{-R_{s_{k}}}>x\right) \leq & \sum_{k=1}^{n} \mathbb{P}\left(X_{k} \mathrm{e}^{-R_{s_{k}}}>x-l(x)\right) \\
& +\sum_{1 \leq i \neq j \leq n} \mathbb{P}\left(X_{i}>\frac{l(x)}{n-1}, X_{j}>\frac{x}{n}\right),
\end{aligned}
$$

where $l(x)$ is defined in (3.9). Since $l(x)$ is infinitely increasing, then, by (3.10), (3.12), (3.7), and (3.8), we have, for all $x \geq \tilde{x}, 0 \leq s_{k} \leq t \leq T$, and $1 \leq k \leq n$,

$$
\begin{aligned}
& \mathbb{P}\left(X_{k} \mathrm{e}^{-R_{s_{k}}}>x-l(x)\right) \\
& =\left(\int_{\delta}^{1}+\int_{0}^{\delta}\right) \bar{F}\left(\frac{x-l(x)}{u}\right) \mathbb{P}\left(\mathrm{e}^{-R_{s_{k}}} \in \mathrm{d} u\right) \\
& \leq \int_{\delta}^{1} \bar{F}\left(\frac{x}{u}-\frac{l(x / u)}{\delta}\right) \mathbb{P}\left(\mathrm{e}^{-R_{s_{k}}} \in \mathrm{d} u\right)+\int_{0}^{\delta} \bar{F}\left(\frac{(1-\delta) x}{u}\right) \mathbb{P}\left(\mathrm{e}^{-R_{s_{k}}} \in \mathrm{d} u\right) \\
& \leq(1+\varepsilon) \int_{\delta}^{1} \bar{F}\left(\frac{x}{u}\right) \mathbb{P}\left(\mathrm{e}^{-R_{s_{k}}} \in \mathrm{d} u\right)+C_{1}(1-\delta)^{-p} \int_{0}^{\delta} \bar{F}\left(\frac{x}{u}\right) \mathbb{P}\left(\mathrm{e}^{-R_{s_{k}}} \in \mathrm{d} u\right) \\
& \leq(1+\varepsilon) \mathbb{P}\left(X_{k} \mathrm{e}^{-R_{s_{k}}}>x\right)+C_{1}(1-\delta)^{-p} \bar{F}\left(\frac{x}{\delta}\right) \mathbb{P}\left(\sup _{s \in[0, T]} R_{s}>-\log \delta\right) \\
& \leq\left(1+\varepsilon+\frac{\varepsilon C_{1}(1-\delta)^{-p}}{1-\varepsilon}\right) \mathbb{P}\left(X_{k} \mathrm{e}^{-R_{s_{k}}}>x\right) .
\end{aligned}
$$

By (3.11) and (3.5), the second sum on the right-hand side of (3.14) can be bounded from above by

$$
\varepsilon \sum_{k=1}^{n} \mathbb{P}\left(X_{k} \mathrm{e}^{-R_{s_{k}}}>x\right)
$$

Combining (3.14)-(3.16), we obtain, for all $x \geq \tilde{x}$ and $0 \leq s_{k} \leq t \leq T, 1 \leq k \leq n$,

$$
\mathbb{P}\left(\sum_{k=1}^{n} X_{k} \mathrm{e}^{-R_{s_{k}}}>x\right) \leq\left(1+\varepsilon\left(2+\frac{C_{1}(1-\delta)^{-p}}{1-\varepsilon}\right)\right) \sum_{k=1}^{n} \mathbb{P}\left(X_{k} \mathrm{e}^{-R_{s_{k}}}>x\right) .
$$

Let $\Delta=\varepsilon\left(2+C_{1}(1-\delta)^{-p}(1-\varepsilon)^{-1}\right)>0$, which can be arbitrarily small because of the arbitrariness of $\varepsilon$. Therefore, (3.6) follows from (3.13) and (3.17).

By (3.4) and (3.6), we have, for all $x \geq \tilde{x}$ (depending only on $F, \Delta$, and $n$ ), $1 \leq n \leq m$, and $t \in \Lambda^{T}$,

$$
\begin{aligned}
(1-\Delta) \sum_{k=1}^{n} \mathbb{P}\left(X_{k} \mathrm{e}^{-R_{\tau_{k}}}>x, N(t)=n\right) & \leq \mathbb{P}\left(\sum_{k=1}^{n} X_{k} \mathrm{e}^{-R_{\tau_{k}}}>x, N(t)=n\right) \\
& \leq(1+\Delta) \sum_{k=1}^{n} \mathbb{P}\left(X_{k} \mathrm{e}^{-R_{\tau_{k}}}>x, N(t)=n\right) .
\end{aligned}
$$

This means that it holds uniformly for all $t \in \Lambda^{T}$ and $1 \leq n \leq m$ that

$$
\mathbb{P}\left(\sum_{k=1}^{n} X_{k} \mathrm{e}^{-R_{\tau_{k}}}>x, N(t)=n\right) \sim \sum_{k=1}^{n} \mathbb{P}\left(X_{k} \mathrm{e}^{-R_{\tau_{k}}}>x, N(t)=n\right) .
$$


Thus, it holds uniformly for all $t \in \Lambda^{T}$ that

$$
\begin{aligned}
I_{1} & \sim \sum_{n=1}^{m} \sum_{k=1}^{n} \mathbb{P}\left(X_{k} \mathrm{e}^{-R_{\tau_{k}}}>x, N(t)=n\right) \\
& =\left(\sum_{n=1}^{\infty}-\sum_{n=m+1}^{\infty}\right) \sum_{k=1}^{n} \mathbb{P}\left(X_{k} \mathrm{e}^{-R_{\tau_{k}}}>x, N(t)=n\right)=: I_{3}-I_{4},
\end{aligned}
$$

where

$$
I_{3}=\sum_{k=1}^{\infty} \mathbb{P}\left(X_{k} \mathrm{e}^{-R_{\tau_{k}}}>x, N(t) \geq k\right)=\int_{0-}^{t} \mathbb{P}\left(X_{1} \mathrm{e}^{-R_{s}}>x\right) \lambda(\mathrm{d} s) .
$$

Since

$$
I_{4} \leq \bar{F}(x) \sum_{n=m+1}^{\infty} n \mathbb{P}(N(t)=n)=\bar{F}(x) \mathbb{E}\left[N(t) \mathbf{1}_{\{N(t)>m\}}\right],
$$

similarly to the proof of (3.3), and by (3.1) and Lemma 3.1, we obtain

$$
\begin{aligned}
\lim _{m \rightarrow \infty} \limsup _{x \rightarrow \infty} \sup _{t \in \Lambda^{T}} \frac{I_{4}}{\int_{0-}^{t} \mathbb{P}\left(X_{1} \mathrm{e}^{-R_{s}}>x\right) \lambda(\mathrm{d} s)} & \leq \frac{1}{C_{2}} \lim _{m \rightarrow \infty} \sup _{t \in \Lambda^{T}} \lambda^{-1}(t) \mathbb{E}\left[N(t) \mathbf{1}_{\{N(t)>m\}}\right] \\
& =0
\end{aligned}
$$

Therefore, by (3.3) and (3.18)-(3.20), (2.1) holds uniformly for all $t \in \Lambda^{T}$, completing the proof.

Proof of Corollary 2.1. The upper bound of $\psi(x, t)$ is trivial. Indeed, by Theorem 2.1, it holds uniformly for all $t \in \Lambda^{T}$ that

$$
\psi(x, t) \leq \mathbb{P}(D(t)>x) \sim \int_{0-}^{t} \mathbb{P}\left(X_{1} \mathrm{e}^{-R_{s}}>x\right) \lambda(\mathrm{d} s) .
$$

We now turn to the asymptotic lower bound of $\psi(x, t)$. For any $0<\varepsilon<1$, since the positive Lévy process $\left\{R_{t}, t \geq 0\right\}$ has nondecreasing paths, and using the fact that $0 \leq c(u) \leq M$ and (2.1), we have, for all $t \in \Lambda^{T}$ and sufficiently large $x$,

$$
\begin{aligned}
\psi(x, t) & \geq \mathbb{P}(D(t)>x+M T) \\
& \geq \mathbb{P}(D(t)>(1+\varepsilon) x) \\
& \sim \int_{0-}^{t} \int_{0}^{1} \bar{F}\left(\frac{(1+\varepsilon) x}{u}\right) \mathbb{P}\left(\mathrm{e}^{-R_{s}} \in \mathrm{d} u\right) \lambda(\mathrm{d} s) \\
& \geq \inf _{u \in(0,1]} \frac{\bar{F}((1+\varepsilon) x / u)}{\bar{F}(x / u)} \int_{0-}^{t} \int_{0}^{1} \bar{F}\left(\frac{x}{u}\right) \mathbb{P}\left(\mathrm{e}^{-R_{s}} \in \mathrm{d} u\right) \lambda(\mathrm{d} s) \\
& \gtrsim \bar{F}_{*}(1+\varepsilon) \int_{0-}^{t} \mathbb{P}\left(X_{1} \mathrm{e}^{-R_{s}}>x\right) \lambda(\mathrm{d} s) .
\end{aligned}
$$

Noting that $\bar{F}_{*}(1+\varepsilon) \rightarrow 1$ as $\varepsilon \downarrow 0$ because $F \in \mathcal{C}$, the desired lower bound follows from (3.22). This completes the proof. 


\section{Proof of Theorem 2.2}

We start this section with a series of lemmas. The first two lemmas are special cases of Lemmas 3.2 and 3.3 of [15] under the independence structure between $\left\{X_{n}, n \geq 1\right\}$ and $\left\{\theta_{n}, n \geq 1\right\}$.

Lemma 4.1. Under the conditions of Theorem 2.2, for each $k \geq 1$, it holds uniformly for all $t \in \Lambda$ that

$$
\bar{F}(x) \mathbb{P}\left(\tau_{k} \leq t\right)=O(1) \mathbb{P}\left(X_{k} \mathrm{e}^{-R_{\tau_{k}}} \mathbf{1}_{\left\{\tau_{k} \leq t\right\}}>x\right) .
$$

Lemma 4.2. Under the conditions of Theorem 2.2, for any positive function $a(x)=x / l(x) \rightarrow$ $\infty$, with the slowly varying function $l(x) \rightarrow \infty$, and each $k \geq 1$, it holds uniformly for all $t \in \Lambda$ that

$$
\mathbb{P}\left(\mathrm{e}^{-R_{\tau_{k}}} \mathbf{1}_{\left\{\tau_{k} \leq t\right\}}>a(x)\right)=o(1) \mathbb{P}\left(X_{k} \mathrm{e}^{-R_{\tau_{k}}} \mathbf{1}_{\left\{\tau_{k} \leq t\right\}}>x\right) .
$$

The next lemma plays an important role in the proof of Theorem 2.2.

Lemma 4.3. Under the conditions of Theorem 2.2, for any fixed $0<y<1$ and each $k \geq 1$, it holds uniformly for all $t \in \Lambda_{\epsilon}$ that

$$
\mathbb{P}\left(X_{k} \mathrm{e}^{-R_{\tau_{k}}} \mathbf{1}_{\left\{\tau_{k} \leq t\right\}}>x y\right) \lesssim y^{-\beta} \mathbb{P}\left(X_{k} \mathrm{e}^{-R_{\tau_{k}}} \mathbf{1}_{\left\{\tau_{k} \leq t\right\}}>x\right) .
$$

Proof. We first show that there exists some $\Delta>0$, not depending on $t$, such that

$$
\mathbb{P}\left(\mathrm{e}^{-R_{\tau_{k}}} \mathbf{1}_{\left\{\tau_{k} \leq t\right\}}>\Delta\right)>0
$$

holds for all $t \in \Lambda_{\epsilon}$. Indeed, if $\mathbb{P}\left(\theta_{1}=0\right)>0$ then, for any $\Delta \in(0,1)$ and all $t \in \Lambda$,

$$
\mathbb{P}\left(\mathrm{e}^{-R_{\tau_{k}}} \mathbf{1}_{\left\{\tau_{k} \leq t\right\}}>\Delta\right) \geq \mathbb{P}\left(\mathrm{e}^{-R_{\tau_{k}}} \mathbf{1}_{\left\{\tau_{k}=0\right\}}>\Delta\right)=\left(\mathbb{P}\left(\theta_{1}=0\right)\right)^{k}>0 .
$$

If $\mathbb{P}\left(\theta_{1}=0\right)=0$ then $\epsilon / k \in \Lambda$. Hence,

$$
\mathbb{P}\left(\tau_{k} \leq \epsilon\right) \geq\left(\mathbb{P}\left(\theta_{1} \leq \frac{\epsilon}{k}\right)\right)^{k}>0 .
$$

For all $t \in \Lambda_{\epsilon}$ and $x \geq 0$,

$$
\begin{aligned}
\mathbb{P}\left(\mathrm{e}^{-R_{\tau_{k}}} \mathbf{1}_{\left\{\tau_{k} \leq t\right\}}>x\right) & \geq \int_{0-}^{\epsilon} \mathbb{P}\left(\mathrm{e}^{-R_{s}}>x\right) \mathbb{P}\left(\tau_{k} \in \mathrm{d} s\right) \\
& \geq \int_{0-}^{\epsilon} \mathbb{P}\left(\sup _{s \in[0, \epsilon]} R_{s}<-\log x\right) \mathbb{P}\left(\tau_{k} \in \mathrm{d} s\right) .
\end{aligned}
$$

Noting that $\mathbb{P}\left(\sup _{[0, \epsilon]} R_{s}=\infty\right)=0$, there exists some $\Delta>0$, not depending on $t$, such that

$$
C_{4}:=\mathbb{P}\left(\sup _{s \in[0, \epsilon]} R_{S}<-\log \Delta\right)>0 .
$$

Substituting this into (4.3), and by (4.2), we have, for all $t \in \Lambda_{\epsilon}$,

$$
\mathbb{P}\left(\mathrm{e}^{-R_{\tau_{k}}} \mathbf{1}_{\left\{\tau_{k} \leq t\right\}}>\Delta\right) \geq C_{4} \mathbb{P}\left(\tau_{k} \leq \epsilon\right) \geq C_{4}\left(\mathbb{P}\left(\theta_{1} \leq \frac{\epsilon}{k}\right)\right)^{k}>0 .
$$


We now prove that

$$
\mathbb{P}\left(\mathrm{e}^{-R_{\tau_{k}}} \mathbf{1}_{\left\{\tau_{k} \leq t\right\}}>x\right)=o(\bar{F}(x))
$$

holds uniformly for all $t \in \Lambda$. Indeed, for all $t \in \Lambda$, by Markov's inequality, $\phi(r)<0$, and Lemma 2.2, we have

$$
\begin{aligned}
\mathbb{P}\left(\mathrm{e}^{-R_{\tau_{k}}} \mathbf{1}_{\left\{\tau_{k} \leq t\right\}}>x\right) & \leq \int_{0-}^{\infty} \mathbb{P}\left(\mathrm{e}^{-R_{s}}>x\right) \mathbb{P}\left(\tau_{k} \in \mathrm{d} s\right) \\
& \leq x^{-r} \int_{0-}^{\infty} \mathrm{e}^{s \phi(r)} \mathbb{P}\left(\tau_{k} \in \mathrm{d} s\right) \\
& =o(\bar{F}(x)) .
\end{aligned}
$$

Claim (4.4) implies that there exists some increasing function $l(x) \uparrow \infty$, not depending on $t$, such that $x / l(x) \rightarrow \infty$ and

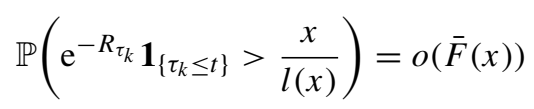

holds uniformly for all $t \in \Lambda$. Then, for all $x>\Delta$, any fixed $y \in(0,1)$, and all $t \in \Lambda_{\epsilon}$,

$$
\begin{aligned}
& \frac{\mathbb{P}\left(X_{k} \mathrm{e}^{-R_{\tau_{k}}} \mathbf{1}_{\left\{\tau_{k} \leq t\right\}}>x y\right)}{\mathbb{P}\left(X_{k} \mathrm{e}^{-R_{\tau_{k}}} \mathbf{1}_{\left\{\tau_{k} \leq t\right\}}>x\right)} \leq \frac{\int_{0-}^{x / l(x)} \bar{F}(x y / u) \mathbb{P}\left(\mathrm{e}^{-R_{\tau_{k}}} \mathbf{1}_{\left\{\tau_{k} \leq t\right\}} \in \mathrm{d} u\right)}{\int_{0-}^{x / l(x)} \bar{F}(x / u) \mathbb{P}\left(\mathrm{e}^{-R_{\tau_{k}}} \mathbf{1}_{\left\{\tau_{k} \leq t\right\}} \in \mathrm{d} u\right)} \\
& +\frac{\int_{x / l(x)}^{\infty} \bar{F}(x y / u) \mathbb{P}\left(\mathrm{e}^{-R_{\tau_{k}}} \mathbf{1}_{\left\{\tau_{k} \leq t\right\}} \in \mathrm{d} u\right)}{\int_{\Delta}^{\infty} \bar{F}(x / u) \mathbb{P}\left(\mathrm{e}^{-R_{\tau_{k}}} \mathbf{1}_{\left\{\tau_{k} \leq t\right\}} \in \mathrm{d} u\right)} \\
& \leq \sup _{z \geq l(x)} \frac{\bar{F}(y z)}{\bar{F}(z)}+\frac{\mathbb{P}\left(\mathrm{e}^{-R_{\tau_{k}}} \mathbf{1}_{\left\{\tau_{k} \leq t\right\}}>x / l(x)\right)}{\bar{F}(x / \Delta) \mathbb{P}\left(\mathrm{e}^{-R_{\tau_{k}}} \mathbf{1}_{\left\{\tau_{k} \leq t\right\}}>\Delta\right)},
\end{aligned}
$$

which, combined with (4.1) and (4.5), leads to the desired result.

Lemma 4.4. Under the conditions of Theorem 2.2, for each $j>i \geq 1$, it holds uniformly for all $t \in \Lambda$ that

$$
\mathbb{P}\left(X_{i} \mathrm{e}^{-R_{\tau_{i}}} \mathbf{1}_{\left\{\tau_{i} \leq t\right\}}>x, X_{j} \mathrm{e}^{-R_{\tau_{j}}} \mathbf{1}_{\left\{\tau_{j} \leq t\right\}}>x\right)=o(1) \sum_{k=i, j} \mathbb{P}\left(X_{k} \mathrm{e}^{-R_{\tau_{k}}} \mathbf{1}_{\left\{\tau_{k} \leq t\right\}}>x\right) .
$$

Proof. By Lemma 4.2, uniformly for all $t \in \Lambda$,

$$
\begin{aligned}
& \mathbb{P}\left(X_{i} \mathrm{e}^{-R_{\tau_{i}}} \mathbf{1}_{\left\{\tau_{i} \leq t\right\}}>x, X_{j} \mathrm{e}^{-R_{\tau_{j}}} \mathbf{1}_{\left\{\tau_{j} \leq t\right\}}>x\right) \\
& \quad \leq \mathbb{P}\left(X_{i} \mathrm{e}^{-R_{\tau_{i}}}>x, X_{j} \mathrm{e}^{-R_{\tau_{j}}}>x, \tau_{i} \leq t, \mathrm{e}^{-R_{\tau_{j}}} \leq a(x)\right)+\mathbb{P}\left(\mathrm{e}^{-R_{\tau_{j}}} \mathbf{1}_{\left\{\tau_{j} \leq t\right\}}>a(x)\right) \\
& \quad=: J_{1}+o(1) \mathbb{P}\left(X_{j} \mathrm{e}^{\left.-R_{\tau_{j}} \mathbf{1}_{\left\{\tau_{j} \leq t\right\}}>x\right),}\right.
\end{aligned}
$$

where $a(x)$ is the function defined in Lemma 4.2. For $J_{1}$, since $\left\{X_{n}, n \geq 1\right\}$ are pairwise NQD, 
it holds uniformly for all $t \in \Lambda$ that

$$
\begin{aligned}
J_{1} & \leq \mathbb{P}\left(X_{i} \mathrm{e}^{-R_{\tau_{i}}}>x, \tau_{i} \leq t, X_{j}>\frac{x}{a(x)}\right) \\
& =\int_{0-}^{t} \int_{0}^{\infty} \mathbb{P}\left(X_{i}>\frac{x}{u}, X_{j}>\frac{x}{a(x)}\right) \mathbb{P}\left(\mathrm{e}^{-R_{s}} \in \mathrm{d} u\right) \mathbb{P}\left(\tau_{i} \in \mathrm{d} s\right) \\
& \leq \int_{0-}^{t} \int_{0}^{\infty} \bar{F}\left(\frac{x}{u}\right) \bar{F}\left(\frac{x}{a(x)}\right) \mathbb{P}\left(\mathrm{e}^{-R_{s}} \in \mathrm{d} u\right) \mathbb{P}\left(\tau_{i} \in \mathrm{d} s\right) \\
& =\bar{F}\left(\frac{x}{a(x)}\right) \mathbb{P}\left(X_{i} \mathrm{e}^{-R_{\tau_{i}}} \mathbf{1}_{\left\{\tau_{i} \leq t\right\}}>x\right) \\
& =o(1) \mathbb{P}\left(X_{i} \mathrm{e}^{\left.-R_{\tau_{i}} \mathbf{1}_{\left\{\tau_{i} \leq t\right\}}>x\right) .}\right.
\end{aligned}
$$

Substituting this estimate into (4.6) completes the proof.

Lemma 4.5. Under the conditions of Theorem 2.2, for each $n \geq 1$, it holds uniformly for all $t \in \Lambda_{\epsilon}$ that

$$
\mathbb{P}\left(\sum_{k=1}^{n} X_{k} \mathrm{e}^{-R_{\tau_{k}}} \mathbf{1}_{\left\{\tau_{k} \leq t\right\}}>x\right) \sim \sum_{k=1}^{n} \mathbb{P}\left(X_{k} \mathrm{e}^{\left.-R_{\tau_{k}} \mathbf{1}_{\left\{\tau_{k} \leq t\right\}}>x\right) .}\right.
$$

Proof. For any $0<\varepsilon<1$, we have

$$
\begin{aligned}
& \mathbb{P}\left(\sum_{k=1}^{n} X_{k} \mathrm{e}^{-R_{\tau_{k}}} \mathbf{1}_{\left\{\tau_{k} \leq t\right\}}>x\right) \\
& \leq \sum_{k=1}^{n} \mathbb{P}\left(X_{k} \mathrm{e}^{-R_{\tau_{k}}} \mathbf{1}_{\left\{\tau_{k} \leq t\right\}}>(1-\varepsilon) x\right) \\
& +\mathbb{P}\left(\sum_{k=1}^{n} X_{k} \mathrm{e}^{-R_{\tau_{k}}} \mathbf{1}_{\left\{\tau_{k} \leq t\right\}}>x, \max _{1 \leq k \leq n} X_{k} \mathrm{e}^{-R_{\tau_{k}}} \mathbf{1}_{\left\{\tau_{k} \leq t\right\}} \leq(1-\varepsilon) x\right) \\
& =: J_{2}+J_{3} \text {. }
\end{aligned}
$$

We first consider $J_{2}$. By Lemma 4.3,

$$
J_{2} \lesssim(1-\varepsilon)^{-\beta} \sum_{k=1}^{n} \mathbb{P}\left(X_{k} \mathrm{e}^{-R_{\tau_{k}}} \mathbf{1}_{\left\{\tau_{k} \leq t\right\}}>x\right)
$$

holds uniformly for all $t \in \Lambda_{\epsilon}$. For $J_{3}$, by Lemmas 4.4 and 4.3,

$$
\begin{aligned}
J_{3} & \leq \mathbb{P}\left(\bigcup _ { i = 1 } ^ { n } \left\{X_{i} \mathrm{e}^{-R_{\tau_{i}}} \mathbf{1}_{\left\{\tau_{i} \leq t\right\}}>\frac{x}{n}, \sum_{1 \leq j \leq n, j \neq i} X_{j} \mathrm{e}^{\left.\left.-R_{\tau_{j}} \mathbf{1}_{\left\{\tau_{j} \leq t\right\}}>\varepsilon x\right\}\right)}\right.\right. \\
& \leq \sum_{i=1}^{n} \sum_{1 \leq j \leq n, j \neq i} \mathbb{P}\left(X_{i} \mathrm{e}^{-R_{\tau_{i}}} \mathbf{1}_{\left\{\tau_{i} \leq t\right\}}>\frac{\varepsilon x}{n}, X_{j} \mathrm{e}^{\left.-R_{\tau_{j}} \mathbf{1}_{\left\{\tau_{j} \leq t\right\}}>\frac{\varepsilon x}{n}\right)}\right. \\
& =o(1) \sum_{i=1}^{n} \mathbb{P}\left(X_{i} \mathrm{e}^{\left.-R_{\tau_{i}} \mathbf{1}_{\left\{\tau_{i} \leq t\right\}}>x\right)}\right.
\end{aligned}
$$


holds uniformly for all $t \in \Lambda_{\epsilon}$. From (4.7)-(4.9), it holds uniformly for all $t \in \Lambda_{\epsilon}$ that

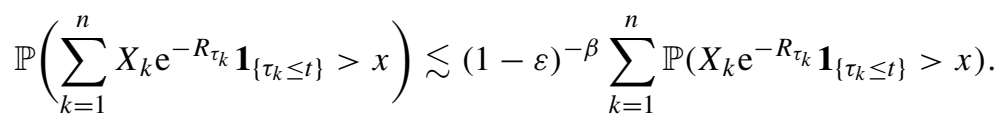

Then the upper bound follows from (4.10) and the arbitrariness of $\varepsilon$.

For the lower bound, according to the Bonferroni inequality,

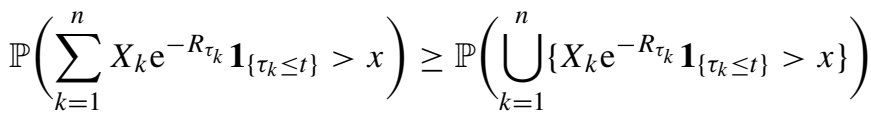

$$
\begin{aligned}
& \geq \sum_{k=1}^{n} \mathbb{P}\left(X_{k} \mathrm{e}^{-R_{\tau_{k}}} \mathbf{1}_{\left\{\tau_{k} \leq t\right\}}>x\right) \\
& -\sum_{1 \leq i<j \leq n} \mathbb{P}\left(X_{i} \mathrm{e}^{-R_{\tau_{i}}} \mathbf{1}_{\left\{\tau_{i} \leq t\right\}}>x, X_{j} \mathrm{e}^{-R_{\tau_{j}}} \mathbf{1}_{\left\{\tau_{j} \leq t\right\}}>x\right) \\
& \sim \sum_{k=1}^{n} \mathbb{P}\left(X_{k} \mathrm{e}^{\left.-R_{\tau_{k}} \mathbf{1}_{\left\{\tau_{k} \leq t\right\}}>x\right)}\right.
\end{aligned}
$$

holds uniformly for all $t \in \Lambda$, where we used Lemma 4.4 in the last step.

Lemma 4.6. ([15].) Under the conditions of Theorem 2.2, it holds that

$$
\begin{aligned}
& \lim _{n \rightarrow \infty} \limsup _{x \rightarrow \infty} \sup _{t \in \Lambda} \frac{\mathbb{P}\left(\sum_{k=n+1}^{\infty} X_{k} \mathrm{e}^{-R_{\tau_{k}}} \mathbf{1}_{\left\{\tau_{k} \leq t\right\}}>x\right)}{\mathbb{P}\left(X_{1} \mathrm{e}^{-R_{\tau_{1}}} \mathbf{1}_{\left\{\tau_{1} \leq t\right\}}>x\right)} \\
& \quad=\lim _{n \rightarrow \infty} \limsup _{x \rightarrow \infty} \sup _{t \in \Lambda} \frac{\sum_{k=n+1}^{\infty} \mathbb{P}\left(X_{k} \mathrm{e}^{\left.-R_{\tau_{k}} \mathbf{1}_{\left\{\tau_{k} \leq t\right\}}>x\right)}\right.}{\mathbb{P}\left(X_{1} \mathrm{e}^{-R_{\tau_{1}}} \mathbf{1}_{\left\{\tau_{1} \leq t\right\}}>x\right)} \\
& \quad=0 .
\end{aligned}
$$

Proof of Theorem 2.2. For any $\varepsilon>0$, according to Lemma 4.6, there exists some sufficiently large integer $n_{0}$ such that, uniformly for all $t \in \Lambda$,

$$
\begin{aligned}
\max & \left\{\mathbb{P}\left(\sum_{k=n_{0}+1}^{\infty} X_{k} \mathrm{e}^{-R_{\tau_{k}}} \mathbf{1}_{\left\{\tau_{k} \leq t\right\}}>x\right), \sum_{k=n_{0}+1}^{\infty} \mathbb{P}\left(X_{k} \mathrm{e}^{-R_{\tau_{k}}} \mathbf{1}_{\left\{\tau_{k} \leq t\right\}}>x\right)\right\} \\
& \lesssim \varepsilon \mathbb{P}\left(X_{1} \mathrm{e}^{-R_{\tau_{1}}} \mathbf{1}_{\left\{\tau_{1} \leq t\right\}}>x\right) .
\end{aligned}
$$

For the upper bound, by Lemma 4.5, (4.11), and Lemma 4.3, for any $0<\delta<1$,

$$
\begin{aligned}
& \mathbb{P}(D(t)>x) \leq \mathbb{P}\left(\sum_{k=1}^{n_{0}} X_{k} \mathrm{e}^{-R_{\tau_{k}}} \mathbf{1}_{\left\{\tau_{k} \leq t\right\}}>(1-\delta) x\right)+\mathbb{P}\left(\sum_{k=n_{0}+1}^{\infty} X_{k} \mathrm{e}^{-R_{\tau_{k}}} \mathbf{1}_{\left\{\tau_{k} \leq t\right\}}>\delta x\right) \\
& \lesssim \sum_{k=1}^{n_{0}} \mathbb{P}\left(X_{k} \mathrm{e}^{-R_{\tau_{k}}} \mathbf{1}_{\left\{\tau_{k} \leq t\right\}}>(1-\delta) x\right)+\varepsilon \mathbb{P}\left(X_{1} \mathrm{e}^{-R_{\tau_{1}}} \mathbf{1}_{\left\{\tau_{1} \leq t\right\}}>\delta x\right) \\
& \lesssim(1-\delta)^{-\beta} \sum_{k=1}^{n_{0}} \mathbb{P}\left(X_{k} \mathrm{e}^{-R_{\tau_{k}}} \mathbf{1}_{\left\{\tau_{k} \leq t\right\}}>x\right)+\varepsilon \delta^{-\beta} \mathbb{P}\left(X_{1} \mathrm{e}^{-R_{\tau_{1}}} \mathbf{1}_{\left\{\tau_{1} \leq t\right\}}>x\right) \\
& \leq\left((1-\delta)^{-\beta}+\varepsilon \delta^{-\beta}\right) \int_{0-}^{t} \mathbb{P}\left(X_{1} \mathrm{e}^{-R_{s}}>x\right) \lambda(\mathrm{d} s)
\end{aligned}
$$


holds uniformly for all $t \in \Lambda_{\epsilon}$. Thus, the upper bound of (2.1) follows from (4.12) by first letting $\varepsilon \downarrow 0$ and then $\delta \downarrow 0$.

For the lower bound, from Lemma 4.5 and (4.11),

$$
\begin{aligned}
\mathbb{P}(D(t)>x) & \geq \mathbb{P}\left(\sum_{k=1}^{n_{0}} X_{k} \mathrm{e}^{-R_{\tau_{k}}} \mathbf{1}_{\left\{\tau_{k} \leq t\right\}}>x\right) \\
& \sim\left(\sum_{k=1}^{\infty}-\sum_{k=n_{0}+1}^{\infty}\right) \mathbb{P}\left(X_{k} \mathrm{e}^{-R_{\tau_{k}}} \mathbf{1}_{\left\{\tau_{k} \leq t\right\}}>x\right) \\
& \geq(1-\varepsilon) \int_{0-}^{t} \mathbb{P}\left(X_{1} \mathrm{e}^{-R_{s}}>x\right) \lambda(\mathrm{d} s)
\end{aligned}
$$

holds uniformly for all $t \in \Lambda_{\epsilon}$. This completes the proof.

Proof of Corollary 2.2. Clearly, Theorem 2.2 implies that the upper bound (3.21) holds uniformly for all $t \in \Lambda_{\epsilon}$.

We estimate the lower bound for the ruin probability. For any $\varepsilon>0$ and the integer $n_{0}$ defined in the proof of Theorem 2.2, we have

$$
\begin{aligned}
\psi(x, t) & \geq \mathbb{P}(D(t)-M Z>x) \\
& \geq \mathbb{P}\left(\sum_{k=1}^{n_{0}} X_{k} \mathrm{e}^{-R_{\tau_{k}}} \mathbf{1}_{\left\{\tau_{k} \leq t\right\}}>(1+\varepsilon) x\right)-\mathbb{P}\left(Z>\frac{\varepsilon x}{M}\right) \\
& =: J_{4}-J_{5},
\end{aligned}
$$

where $Z=\int_{0-}^{\infty} \mathrm{e}^{-R_{s}} \mathrm{~d} s$ and $M>0$ is the upper bound of the intensity of premium payments. As in (4.13), by Lemmas 4.5 and 4.3 and (4.11), it follows that

$$
\begin{aligned}
J_{4} & \gtrsim \sum_{k=1}^{n_{0}} \mathbb{P}\left(X_{k} \mathrm{e}^{-R_{\tau_{k}}} \mathbf{1}_{\left\{\tau_{k} \leq t\right\}}>(1+\varepsilon) x\right) \\
& \gtrsim(1+\varepsilon)^{-\beta} \sum_{k=1}^{n_{0}} \mathbb{P}\left(X_{k} \mathrm{e}^{-R_{\tau_{k}}} \mathbf{1}_{\left\{\tau_{k} \leq t\right\}}>x\right) \\
& \geq(1+\varepsilon)^{-\beta}(1-\varepsilon) \int_{0-}^{t} \mathbb{P}\left(X_{1} \mathrm{e}^{-R_{s}}>x\right) \lambda(\mathrm{d} s)
\end{aligned}
$$

holds uniformly for all $t \in \Lambda_{\epsilon}$. For $J_{5}$, by Markov's inequality and Lemmas 3.2 and 2.2, we have

$$
J_{5} \leq\left(\frac{M}{\varepsilon}\right)^{r} \mathbb{E}\left[Z^{r}\right] x^{-r}=o(\bar{F}(x)) .
$$

If $\mathbb{P}\left(\theta_{1}=0\right)>0$ then, by Lemma 4.1 , it holds that, uniformly for all $t \in \Lambda$,

$$
\bar{F}(x) \leq\left(\mathbb{P}\left(\theta_{1}=0\right)\right)^{-1} \bar{F}(x) \mathbb{P}\left(\theta_{1} \leq t\right)=O(1) \mathbb{P}\left(X_{1} \mathrm{e}^{-R_{\tau_{1}}} \mathbf{1}_{\left\{\tau_{1} \leq t\right\}}>x\right),
$$

which, together with (4.16), yields

$$
J_{5}=o(1) \mathbb{P}\left(X_{1} \mathrm{e}^{-R_{\tau_{1}}} \mathbf{1}_{\left\{\tau_{1} \leq t\right\}}>x\right)=o(1) \int_{0-}^{t} \mathbb{P}\left(X_{1} \mathrm{e}^{-R_{s}}>x\right) \lambda(\mathrm{d} s)
$$


uniformly for all $t \in \Lambda$. Similarly, if $\mathbb{P}\left(\theta_{1}=0\right)=0$, also by Lemma 4.1, it holds uniformly for all $t \in \Lambda_{\epsilon}$ that

$$
\bar{F}(x) \leq\left(\mathbb{P}\left(\theta_{1} \leq \epsilon\right)\right)^{-1} \bar{F}(x) \mathbb{P}\left(\theta_{1} \leq t\right)=O(1) \mathbb{P}\left(X_{1} \mathrm{e}^{-R_{\tau_{1}}} \mathbf{1}_{\left\{\tau_{1} \leq t\right\}}>x\right),
$$

which implies that (4.17) holds uniformly for all $t \in \Lambda_{\epsilon}$. Combining (4.14)-(4.17) and noting the arbitrariness of $\varepsilon$, we derive the lower bound.

\section{Acknowledgements}

The authors are most grateful to the anonymous referee for her/his very thorough reading of the paper and valuable suggestions, which greatly improved the original results and presentation. The first and second authors were supported by the National Natural Science Foundation of China (grant numbers 11001052 and 11226211).

\section{References}

[1] Asmussen, S. (1998). Subexponential asymptotics for stochastic processes: extremal behavior, stationary distributions and first passage probabilities. Ann. Appl. Prob. 8, 354-374.

[2] Bingham, N. H., Goldie, C. M. And Teugels, J. L. (1987). Regular Variation. Cambridge University Press.

[3] Block, H. W., Savits, T. H. and Shaked, M. (1982). Some concepts of negative dependence. Ann. Prob. 10, $765-772$.

[4] CAI, J. (2004). Ruin probabilities and penalty functions with stochastic rates of interest. Stoch. Process. Appl. 112, 53-78.

[5] Chen, Y. AND NG, K. W. (2007). The ruin probability of the renewal model with constant interest force and negatively dependent heavy-tailed claims. Insurance Math. Econom. 40, 415-423.

[6] Cline, D. B. H. and Samorodnitsky, G. (1994). Subexponentiality of the product of independent random variables. Stoch. Process. Appl. 49, 75-98.

[7] Cont, R. and Tankov, P. (2004). Financial Modelling with Jump Processes. Chapman \& Hall/CRC, Boca Raton, FL.

[8] HaO, X. AND TANG, Q. (2008). A uniform asymptotic estimate for discounted aggregate claims with subexponential tails. Insurance Math. Econom. 43, 116-120.

[9] Johg-Dev, K. and Proschan, F. (1983). Negative association of random variables, with applications. Ann. Statist. 11, 286-295.

[10] Kalashnikov, V. and Konstantinides, D. (2000). Ruin under interest force and subexponential claims: a simple treatment. Insurance Math. Econom. 27, 145-149.

[11] Kalashnikov, V. And Norberg, R. (2002). Power tailed ruin probabilities in the presence of risky investments. Stoch. Process. Appl. 98, 211-228.

[12] Klüppelberg, C. And Stadtmüller, U. (1998). Ruin probabilities in the presence of heavy-tails and interest rates. Scand. Actuarial J. 1998, 49-58.

[13] Konstantinides, D., Tang, Q. and Tsitsiashvili, G. (2002). Estimates for the ruin probability in the classical risk model with constant interest force in the presence of heavy tails. Insurance Math. Econom. 31, 447-460.

[14] Lehmann, E. L. (1966). Some concepts of dependence. Ann. Math. Statist. 37, 1137-1153.

[15] LI, J. (2012). Asymptotics in a time-dependent renewal risk model with stochastic return. J. Math. Anal. Appl. 387, 1009-1023.

[16] Maulik, K. And Resnick, S. (2004). Characterizations and examples of hidden regular variation. Extremes 7, 31-67.

[17] Maulik, K. and Zwart, B. (2006). Tail asymptotics for exponential functionals of Lévy processes. Stoch. Process. Appl. 116, 156-177.

[18] Paulsen, J. (1993). Risk theory in a stochastic economic environment. Stoch. Process. Appl. 46, 327-361.

[19] Paulsen, J. (2002). On Cramér-like asymptotics for risk processes with stochastic return on investments. Ann. Appl. Prob. 12, 1247-1260.

[20] Paulsen, J. and Gjessing, H. K. (1997). Ruin theory with stochastic return on investments. Adv. Appl. Prob. 29, 965-985.

[21] TANG, Q. (2005). The finite time ruin probability of the compound Poisson model with constant interest force. J. Appl. Prob. 42, 608-619.

[22] TAng, Q. (2007). Heavy tails of discounted aggregate claims in the continuous-time renewal model. J. Appl. Prob. 44, 285-294. 
[23] Tang, Q. And Tsitsiashvili, G. (2003). Precise estimates for the ruin probability in finite horizon in a discretetime model with heavy-tailed insurance and financial risks. Stoch. Process. Appl. 108, 299-325.

[24] TANG, Q., WANG, G. AND YuEN, K. C. (2010). Uniform tail asymptotics for the stochastic present value of aggregate claims in the renewal risk model. Insurance Math. Econom. 46, 362-370.

[25] YANG, Y. AND WANG, Y. (2010). Asymptotics for ruin probability of some negatively dependent risk models with a constant interest rate and dominatedly-varying-tailed claims. Statist. Prob. Lett. 80, 143-154.

[26] YUEN, K. C., WANG, G. AND NG, K. W. (2004). Ruin probabilities for a risk process with stochastic return on investments. Stoch. Process. Appl. 110, 259-274.

[27] Yuen, K. C., Wang, G. And Wu, R. (2006). On the renewal risk process with stochastic interest. Stoch. Process. Appl. 116, 1496-1510. 